\title{
The time course of sociolinguistic influences on wordlikeness judgments
}

James Myers, Tsung-Ying Chen

Graduate Institute of Linguistics, National Chung Cheng University, Taiwan

https://doi.org/10.36505/ExLing-2016/07/0026/000285

\begin{abstract}
This study examined how and when sociolinguistic factors affect wordlikeness judgments by near-native bilinguals of Mandarin, the prestige language of Taiwan, and Southern Min (Taiwanese). Auditory syllables nonlexical in both languages were recorded by two bilingual speakers, one with a S. Min accent and one with a Mandarin accent. Accent and target language (judging the syllables as Mandarin-like or as S. Minlike) were crossed across participant groups. Binary judgments collected via the Worldlikeness Web app were analyzed in terms of target language, accent, participant gender, Mandarin and S. Min neighbourhood density, and reaction time. Response patterns were affected by all of these variables, including reaction time, in ways consistent with the differing social status of the two languages.
\end{abstract}

Key words: wordlikeness, neighbourhood density, bilingualism, gender, time course

\section{Introduction}

Mandarin is the prestige language in Taiwan, though many speakers are also native speakers of Southern Min (Taiwanese), another Sinitic language, even if, as adults, they may be more fluent in Mandarin. This social situation raises psycholinguistic questions: how and when is the phonological processing of near-native bilinguals affected by sociolinguistic variables like language status, gender (given that women are expected to favour the prestige norm; Labov, 2001) and accent (given that S. Min-accented Mandarin is expected to be disfavoured; Chung, 2006)?

To find out, we conducted a wordlikeness judgment task in which speakers rated the acceptability of nonlexical items as possible words in Mandarin or in Southern Min. Since this task is sensitive to neighbourhood density (the number of lexical items minimally different from a test item; Bailey and Hahn, 2001), and the influence of neighbourhood density increases over time (Stockall, Stringfellow, and Marantz 2004), we were also interested to see how the social variables interacted with neighbourhood density (including in the non-target language: Frisch and Brea-Spahn 2010), as modulated by reaction time (since slower responses may be sensitive to later processes).

ExLing 2016: Proceedings of $7^{\text {th }}$ Tutorial and Research Workshop on Experimental Linguistics, 27 June - 2 July 2016, Saint Petersburg, Russia 
The lexicons of Mandarin and S. Min share crucial similarities: most morphemes are cognates across these languages, morphemes are virtually always monosyllabic, and syllable structure is very simple. However, the phonotactics of S. Min is less restricted (e.g., licensing an oral/nasal vowel contrast), making its syllable inventory (around 2400) larger than that for Mandarin (around 1400). These similarities and differences make it possible to test bilinguals with a single set of nonlexical syllables that vary in wordlikeness relative to Mandarin, S. Min, or both.

\section{Methods}

We used an auditory wordlikeness judgment task.

Participants. 80 bilingual speakers of Mandarin and Southern Min (mean age 22 years, 42 female) were paid for their participation, with 20 in each of four groups defined by crossing target language and stimulus accent (explained below).

Materials. An initial set of 5116 syllables was generated by randomly combining Mandarin and S. Min onsets and rimes and removing items lexical in either language. IPA transcriptions of these syllables were then presented randomly to two female bilingual speakers for recording, one raised in a Mandarin-speaking home and the other in a S. Min-speaking home, which affected their accents. In a pretest, the sound files were presented to 12 bilingual listeners. Items that were misperceived as lexical by more than one listener were reviewed by another two bilingual speakers and removed if the speakers agreed on the judgment. This screening procedure left 129 syllable types for the main experiment. Mandarin and S. Min neighbourhood densities were computed for each item; to further reduce the influence of acoustic ambiguity and to aid crosslinguistic comparisons, these computations ignored tone and vowel nasality. Neighbourhood densities were correlated $\left(r^{2}(127)=.1, p<.001\right)$, but not enough to pose collinearity problems.

Procedure. Depending on which of the four groups they were assigned to, participants were asked to judge syllables that were or were not S. Min-accented as being like Mandarin or like S. Min. The Web app Worldlikeness (Chen and Myers forthcoming; http://lngproc4083.nitrouspro.com:3000/) was used to present the stimuli in a different random order for each participant. Responses were made by pressing either the ' $L$ ' key (like the target language) or the 'S' key (not like it). Trials ended if a response was received, or else after 4,000 ms. Both responses and reaction times (RT) from stimulus onset were recorded. Experimental parameters and results are available for download from the Worldlikeness website. 


\section{Results and discussion}

The data were analyzed using mixed-effects logistic regression with participant and item as random variables, and with participant gender, target language, stimulus accent, $\log$ Mandarin and S. Min neighbourhood density z scores, log trial RT z scores, and all interactions (except between the two neighbourhood densities) as fixed variables. All of these factors influenced responses in ways consistent with the greater social status of Mandarin (all effects and interactions reported below were significant at $p<.05)$. The overall acceptance rate for Mandarin (.27) was lower than for S. Min (.43), suggesting a greater resistance to nonwords in the more prestigious language. Mandarin neighbours improved Mandarin-likeness judgments, but this factor had no effect on S. Min-likeness. By contrast, S. Min neighbours increased S. Min-likeness but also lowered Mandarin-likeness, as if items were "tainted" by an affinity with the non-prestige language. This negative effect was particularly strong for female participants, reflecting the common finding that women favour prestige norms. S. Min accent also enhanced the positive effect of S. Min neighbours on judgments, but had no effect on the influence of Mandarin neighbours, suggesting that the S. Min lexicon may be encoded less abstractly than the prestige language, making phonetic detail (accent) matter when activating neighbours.

Particularly intriguing were interactions with RT. As shown in Figure 1, when judging Mandarin, slower responses were more accepting (thin lines), while the reverse was true when judging S. Min (thick lines). The left plot in Figure 1 shows that the rise in acceptance was particularly steep when judging the Mandarin-likeness of items with fewer Mandarin neighbours; S. Min-likeness and S. Min neighbours (right plot) showed no such interactions with RT. One interpretation of these results is that Mandarin neighbours are activated quickly but undergo further processing, compared with a more uniform process for S. Min. This may explain why Mandarin neighbours enhance the fastest judgments, yet reverse their influence for slower judgments, perhaps as more distant neighbours become activated. By contrast, the only temporal change for $\mathrm{S}$. Min seems to be a reduction in an initial positive response bias.

\section{Conclusions}

Our bilingual wordlikeness judgment study confirms that gender, accent, and the social status of languages all influence real-time phonological processing. In particular, judgments for the more prestigious language were more critical, were hurt by neighbours in the less prestigious language (especially for women), and may have been processed more 
deeply (perhaps an indirect effect of the participants' lower fluency in the less prestigious language). Many speakers across the world are nearnative bilinguals in languages differing in social prestige. Since our experiment was run using Worldlikeness, a free web app for collecting and sharing wordlikeness judgments, we hope that interested scholars will use it to extend our findings across a much wider variety of languages.
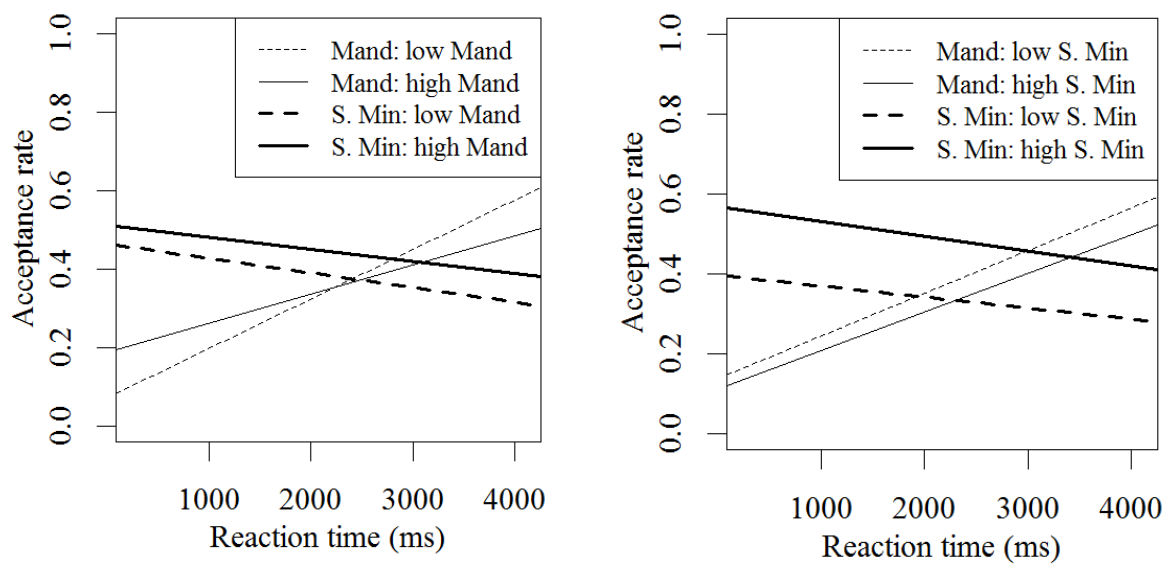

Figure 1. The effects of target language, reaction time, and neighbourhood density (left: Mandarin, right: S. Min) on wordlikeness judgments.

\section{Acknowledgements}

This study was supported by Ministry of Science and Technology (Taiwan) grant MOST 103-2410-H-194-119-MY3.

\section{References}

Bailey, T. M. and Hahn, U. 2001. Determinants of wordlikeness: Phonotactics or lexical neighborhoods? Journal of Memory and Language 44, 568-591.

Chen, T.-Y. and Myers, J. Forthcoming. Worldlikeness: A Web-based tool for typological psycholinguistic research. Proc. of the 40th Annual Penn Ling. Conf., Philadelphia, USA.

Chung, K. S. 2006. Hypercorrection in Taiwan Mandarin. Journal of Asian Pacific Communication 16, 197-214.

Frisch, S. A. and Brea-Spahn, M. R. 2010. Metalinguistic judgments of phonotactics by monolinguals and bilinguals. Laboratory Phonology 1, 345-360.

Labov, W. 2001. Principles of Linguistic Change, vol. 2: Social Factors. Oxford, Blackwell.

Stockall, L., Stringfellow, A., and Marantz, A. 2004. The precise time course of lexical activation: MEG measurements of the effects of frequency, probability, and density in lexical decision. Brain \& Language 90, 88-94. 